\title{
ON POINTED HOPF ALGEBRAS OF DIMENSION $p^{5}$
}

\author{
MATÍAS GRAÑ** \\ Universidad de Buenos Aires, Facultad de Ciencias Exactas y Naturales (1428) Ciudad Universitaria, \\ Buenos Aires, Argentina \\ e-mail:matiasg@dm.uba.ar
}

(Received 18 December, 1998)

\begin{abstract}
We describe all possible coradically graded pointed Hopf algebras of dimension $p^{5}$ (where $p$ is an odd prime number) over an algebraically closed field of characteristic 0 .
\end{abstract}

1991 Mathematics Subject Classification. 16W30.

1. Introduction. The lifting procedure described in [2] is a powerful tool for classifying pointed Hopf algebras. It has been applied successfully to the classification of pointed Hopf algebras of dimension $p^{3}$ in [2] and dimension $p^{4}$ in [4]. It has been used also in the classification of pointed Hopf algebras of dimension 32 in [10]. We describe here all pointed coradically graded Hopf algebras of dimension $p^{5}$ (we assume $p$ is odd since the case $p=2$ is treated in [10]). Some of these algebras are known and can be found in the referred articles as well as in [3], [8]. Classification problems of pointed Hopf algebras have been also treated in [6], [9] and [7].

Our main references for Hopf algebras are [13] and [11]. For Nichols algebras we refer to [12] and [1].

The article is organized as follows: in Section 2 we give the notation and definitions we use and the first results we need. In Section 3 we describe all possible Nichols algebras of dimension $p^{5-j}$ over groups of order $p^{j}(j=1, \ldots, 4)$. In Section 4 we prove necessary auxiliary results; some of them have interest on their own, e.g. Theorem 4.3. In Section 5 we prove that any pointed Hopf algebra of dimension $p^{5}$ over $\mathbf{k}$ is generated by group-like and skew-primitive elements. In other words, any coradically graded pointed Hopf algebra of dimension $p^{5}$ can be recovered by bosonization (or biproduct) from one of the Nichols algebras appearing in Theorem 3.2. Furthermore, this proves also that any pointed Hopf algebra of dimension $p^{5}$ can be recovered by lifting (in the sense of [2]) of one of these bosonizations. Thus the classification of the pointed Hopf algebras of dimension $p^{5}$ could be done in principle using the lifting procedure. This article contains the first steps in this direction. In Section 6 we address the remaining steps and consider some illustrating examples.

This is part of the doctoral thesis of the author, who thanks N. Andruskiewitsch for his guidance.

2. Notation and preliminary results. The letter $\mathbf{k}$ will stand for an algebraically closed field of characteristic 0. All Hopf algebras are k-algebras. For $\Gamma$ a group and $g \in \Gamma$ we denote by $\Gamma_{g}$ the isotropy subgroup $\Gamma_{g}=\{h \in \Gamma \mid h g=g h\}$. Let $q \in \mathbf{k}$. For $n \geq m \in \mathbb{N}$, we use the standard notation

* This work was partially supported by CONICET, CONICOR and SeCyT-UNC. 


$$
(n)_{q}=\sum_{i=0}^{n-1} q^{i},(0)_{q}=1 ; \quad(n)_{q}^{!}=\prod_{i=1}^{n}(i)_{q} ; \quad\left(\begin{array}{c}
n \\
m
\end{array}\right)_{q}=\frac{(n)_{q}^{!}}{(m)_{q}^{!}(n-m)_{q}^{!}} .
$$

For $A$ a Hopf algebra, we say that $A$ is pointed if and only if the simple subcoalgebras of $A$ are 1-dimensional (if and only if the irreducible representations of $A^{*}$ are 1-dimensional).

Let $A=\oplus_{i \geq 0} A(i)$ be a graded Hopf algebra. We say that $A$ is coradically graded if the graduation corresponds to the coradical filtration of $A$; i.e. if $A_{r}=\oplus_{i=0}^{r} A(i)$ $\forall r \geq 0$, where $A_{0} \subseteq A_{1} \subseteq \ldots$ stands for the coradical filtration of $A$. In particular, $A$ being coradically graded and pointed implies that $A(0) \simeq \mathbf{k} \Gamma$, where $\Gamma$ is the group of group-likes of $A$.

Let $H$ be a Hopf algebra. We denote by ${ }_{H}^{H} \mathcal{Y D}$ the category of (left-left) YetterDrinfeld modules over $H$ (see [11]) and by $c$ its braiding. Let $A$ be a coradically graded pointed Hopf algebra and $A(0)=\mathbf{k} \Gamma$; then

$$
R=A^{\mathrm{co} A(0)}=\{x \in A \mid(\mathrm{id} \otimes \pi) \Delta(x)=x \otimes 1\}=\oplus_{i} R(i),
$$

(where $\pi: A \rightarrow A(0)$ is the canonical projection), is a braided Hopf algebra in the category ${ }_{\mathbf{k} \Gamma}^{\mathbf{k}} \mathcal{Y} \mathcal{D}$. The Hopf algebra $A$ can be recovered by bosonization: $A=R \# \mathbf{k} \Gamma$. Furthermore, $R$ is coradically graded and $R(0)=\mathbf{k} 1$. If moreover $R$ is generated as an algebra by $R(1)$, then we say that $R$ is a Nichols algebra.

If $R$ is a Nichols algebra, then $R$ is uniquely determined (up to isomorphism) by $V=R(1)$, which coincides with the space of primitive elements $\mathcal{P}(R)$. We write $R=\mathfrak{B}(V)$.

We refer to the survey [1] for details on these constructions (Nichols algebras are called TOBAs in that article).

Proposition 2.2. Let $\mathbf{f}$ be any field, and let $H$ be a Hopf algebra over $\mathbf{f}$. Let $V$ be an object in ${ }_{H}^{H} \mathcal{Y} \mathcal{D}$. Suppose $V$ has a basis $\left\{x_{1}, \ldots, x_{\theta}\right\}$ such that $c\left(x_{i} \otimes x_{j}\right)=b_{i j} x_{j} \otimes x_{i}$ for certain $b_{i j} \in \mathbf{f}$ ( since $c$ is an automorphism, $b_{i j} \in \mathbf{f}^{\times}$). We take for each $i=1, \ldots, \theta$

$$
N_{i}= \begin{cases}\text { order of } b_{i i} & \text { if } b_{i i} \neq 1 \text { and is a root of unity, } \\ \infty & \text { if } b_{i i} \text { is not a root of unity, } \\ \infty & \text { if } b_{i i}=1 \text { and char } \mathbf{f}=0 \\ \text { char } \mathbf{f} & \text { if } b_{i i}=1 \text { and char } \mathbf{f}>0\end{cases}
$$

Then $\operatorname{dim} \mathfrak{B}(V) \geq \prod_{i} N_{i}$. Moreover, if $\mathfrak{B}(V)$ is finite dimensional, then the equality holds if and only if $b_{i j} b_{j i}=1, \forall i \neq j$.

Proof. See [2, §3].

We recall (see for instance [1]) that if $\Gamma$ is a finite group, the category $\mathbf{k}_{\mathbf{k} \Gamma} \mathcal{Y} \mathcal{D}$ is semisimple. The simple objects are the modules $M(g, \rho)$ defined as follows: let $g \in \Gamma$, $\rho$ an irreducible representation of the isotropy group $\Gamma_{g}$. Let $W$ be the space affording $\rho$, and take

$$
M(g, \rho)=\operatorname{Ind}_{\Gamma_{g}}^{\Gamma} W=\mathbf{k} \Gamma \otimes_{\mathbf{k} \Gamma_{g}} W,
$$

with the usual module structure and the comodule structure given by 


$$
\delta(h \otimes w)=h g h^{-1} \otimes(h \otimes w) \in \mathbf{k} \Gamma \otimes M(g, \rho) .
$$

REMARK 2.3. Since $g$ is central in $\Gamma_{g}$, if $\rho$ is an irreducible representation of $\Gamma_{g}$ then the Schur lemma says that $\rho(g)=q$ id, for some $q \in \mathbf{k}^{\times}$.

Definition 2.4. We say that $V \in{ }_{H}^{H} \mathcal{Y D}$ has a matrix $\left(b_{i j}\right)$ if it has a basis $\left\{x_{1}, \ldots, x_{\theta}\right\}$ such that $c\left(x_{i} \otimes x_{j}\right)=b_{i j} x_{j} \otimes x_{i}$.

This happens for instance if $\Gamma$ is abelian. This happens also under a weaker condition: let $V=\oplus_{i} M\left(g_{i}, \rho_{i}\right)$ and suppose that the subgroup $\Gamma^{\prime}$ of $\Gamma$ generated by the conjugacy classes of all the $g_{i}$ is abelian. Then $V$ comes from the abelian case in the sense of [1, Definition 3.1.8] and consequently has a matrix. In this case $V$ can be considered as a Yetter-Drinfeld module over $\Gamma^{\prime}$ and $\mathfrak{B}(V) \# \mathbf{k} \Gamma$ can be reconstructed as an extension of $\Gamma / \Gamma^{\prime}$ by $\mathfrak{B}(V) \# \mathbf{k} \Gamma^{\prime}$. A sufficient condition for $\Gamma^{\prime}$ to be abelian in the case $V=M(g, \rho)$ is that the isotropy subgroup $\Gamma_{g}$ be invariant in $\Gamma$ (see $[\mathbf{1}$, Lemma 3.1.9]). Since we are working in characteristic 0 , if $V$ has a matrix $\left(b_{i j}\right)$ and $\mathfrak{B}(V)$ is finite dimensional then, by Proposition $2.2, b_{i i} \neq 1, \forall i$.

If $V$ has a matrix $\left(b_{i j}\right)$ with $b_{i j} b_{j i}=1, \forall i \neq j$, then it can be shown that $\mathfrak{B}(V)$ has a PBW basis of the form

$$
\left\{x_{1}^{n_{1}} \cdots x_{\theta}^{n_{\theta}} \mid 0 \leq n_{i}<N_{i}\right\}
$$

where $N_{i}$ is defined as in Proposition 2.2. The relations are given by

$$
x_{i}^{N_{i}}=0, \quad x_{i} x_{j}=b_{i j} x_{j} x_{i}, \forall i>j .
$$

Thus $\mathfrak{B}(V)$ is a quantum linear space as an algebra. We notice that the lines $\mathbf{k} x_{i}$ $(i=1, \ldots, \theta)$ are not Yetter-Drinfeld submodules in general. In order to agree with the terminology of [2], we shall denote such an algebra by QLS only when the lines $\mathbf{k} x_{i}$ are Yetter-Drinfeld modules $\forall i$. Thus, a QLS in ${ }_{\mathbf{k} \Gamma} \mathcal{Y} \mathcal{D}$ is given by a module $V=\oplus_{i=1}^{\theta} M\left(g_{i}, \chi_{i}\right)$, where

$$
\left\{\begin{array}{l}
g_{1}, \ldots, g_{\theta} \in \Gamma \text { are central elements, and } \\
\chi_{1}, \ldots, \chi_{\theta} \in \hat{\Gamma} \text { are characters such that } \\
\chi_{i}\left(g_{j}\right) \chi_{j}\left(g_{i}\right)=1, \quad \forall i \neq j .
\end{array}\right.
$$

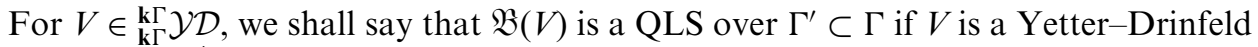
module in ${ }_{\mathbf{k} \Gamma^{\prime}}^{\mathbf{k}} \mathcal{Y} \mathcal{Y}$ and the conditions 2.5 hold for $\Gamma^{\prime}$. A 1-dimensional QLS will be called also Quantum Line (or QL), and a 2-dimensional QLS will be called also Quantum Plane (or QP).

According to [3], if $V$ has a matrix $\left(b_{i j}\right)$ we say that $V$ is of Cartan type if there exists a (generalized) Cartan matrix $\left(a_{i j}\right)$ such that

$$
b_{i j} b_{j i}=b_{i i}^{a_{i j}}, \quad \forall i, j=1, \ldots, \theta .
$$

We transfer to $V$ the terminology over the Cartan matrix $\left(a_{i j}\right)$.

Lemma 2.6. Let $g$ be central in $\Gamma$ and $\rho$ an irreducible representation of $\Gamma$. Let $V=M(g, \rho)$. By 2.3, $g$ acts by a scalar on $V$, say $q$. Let $N$ be the order of $q$. Then $\operatorname{dim} \mathfrak{B}(V) \geq N^{\operatorname{deg} \rho}$. 
Proof. Since $g$ is central, $V$ comes from the abelian case, and consequently $c$ has a matrix $\left(b_{i j}\right)$. It is straightforward to see that $b_{i j}=q, \forall i, j$. Then Proposition 2.2 applies and the result follows.

Lemma 2.7. Let $g \in \Gamma$ and $\rho$ an irreducible representation of $\Gamma_{g}$. Let $V=M(g, \rho)$. Suppose that $\operatorname{dim} V<p$, where $p$ is the smallest prime dividing $|\Gamma|$. Then $g$ is central, $\operatorname{deg} \rho=1$ and thus $\mathfrak{B}(V)$ is a $Q L$ over $\Gamma$ with $\operatorname{dim} \mathfrak{B}(V)=N$, where $N$ is the order of $\rho(g)$.

Proof. We have $\operatorname{dim} V=\left[\Gamma: \Gamma_{g}\right] \operatorname{deg}(\rho)<p$. Since $\left[\Gamma: \Gamma_{g}\right]$ and $\operatorname{deg}(\rho)$ both divide $|\Gamma|$, necessarily $\left[\Gamma: \Gamma_{g}\right]=1$, whence $g$ is central, and $\operatorname{deg}(\rho)=1$. The result follows from Proposition 2.2.

REMARK 2.8. Since $\operatorname{dim} \mathfrak{B}(V) \geq 1+\operatorname{dim} V$, the hypothesis of the preceding lemma is satisfied if $\operatorname{dim} \mathfrak{B}(V) \leq p$.

REMARK 2.9. By Lemma 2.7, we have that if $V=\oplus_{i} M\left(g_{i}, \rho_{i}\right)$ is such that $\operatorname{dim} V<p$, then $g_{i}$ is central and $\rho_{i}$ is a character $\forall i$, and furthermore $\operatorname{dim} \mathfrak{B}(V) \geq \prod_{i} N_{i}$, where $N_{i}$ is the order of $\rho_{i}\left(g_{i}\right)$.

\section{Main results.}

Lemma 3.1. Let $A$ be a coradically graded pointed Hopf algebra of dimension $p^{5}$.

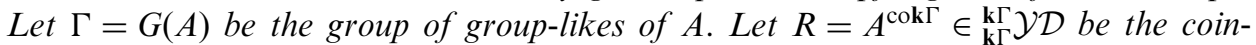
variants (thus $A=R \# \boldsymbol{k} \Gamma$ ) and let $V=R(1)$ be the primitive elements of $R$. Assume that $V$ generates $R$ as an algebra (i.e. $R=\mathfrak{B}(V)$ ). Then the following possibilities arise.

1. If $|\Gamma|=p^{5}$, then $V=0$ and $A=\mathbf{k} \Gamma$.

2. If $|\Gamma|=p^{4}$, then $V$ is 1-dimensional and $R$ is a $Q L S$.

3. If $|\Gamma|=p^{3}$, then $V$ may be 1 or 2-dimensional and $R$ is a $Q L S$ over some subgroup $\Gamma^{\prime}$ of $\Gamma$.

4. If $\Gamma=C_{p} \times C_{p}$, then $V$ is 2-dimensional (and then $R$ is a twisting of a Nichols algebra of type $A_{2}$ ) or $V$ is 3-dimensional (and $R$ is a $Q L S$ ).

5. If $\Gamma=C_{p^{2}}$, then $V$ is 2-dimensional (and in this case $R$ is a $Q L S$ or a twisting of a Nichols algebra of type $A_{2}$ ) or $V$ is 3-dimensional (and $R$ is a $Q L S$ ).

6. If $\Gamma=C_{p}$, then either $V$ is 2-dimensional, $R$ is of type $B_{2}$ and necessarily $p \equiv 1 \bmod 4$, or $V$ is 3-dimensional, $R$ is of type $A_{2} \times A_{1}$ and $p=3$.

Proof. We prove that $\mathfrak{B}(V)$ is of the form claimed.

1. This is immediate.

2. By Remark 2.9 we have $\operatorname{dim} V=1, V=(x)=M(g, \chi), g \in Z(\Gamma)$. Furthermore, $\chi(g)=q$ is such that $q^{p}=1$ (and $q \neq 1$ since $A$ is finite dimensional), whence the structure of $R$ is given by

$$
\begin{gathered}
x^{p}=0, \quad \varepsilon(x)=0, \\
\Delta\left(x^{r}\right)=\sum_{i=0}^{r}\left(\begin{array}{l}
r \\
i
\end{array}\right)_{q} x^{i} \otimes x^{r-i},
\end{gathered}
$$




$$
\delta(x)=g \otimes x, \quad h \rightarrow x=\chi(h) x .
$$

Let $a=x \# 1 \in A$. Then $A$ is generated by $\Gamma$ and $a$, with the structure given by

$$
\begin{gathered}
a^{p}=0, \quad \varepsilon(a)=0, \quad h a h^{-1}=\chi(h) a \quad \forall h \in \Gamma, \\
\Delta\left(a^{r}\right)=\sum_{i=0}^{r}\left(\begin{array}{l}
r \\
i
\end{array}\right)_{q}\left(a^{i} g^{r-i}\right) \otimes a^{r-i} .
\end{gathered}
$$

3. The bound $\operatorname{dim} V \leq 2$ is a consequence of 4.3 below. If $V$ is 1-dimensional, then $V=M(g, \chi)$ and $A$ is given exactly as in the case $|\Gamma|=p^{4}$ with the only exception being that $q$ has order $p^{2}$ and the relation on $a$ is $a^{p^{2}}=0$. If $V$ is 2-dimensional, [1, Proposition 3.1.11] applies and $V$ comes from the abelian case; i.e. $V$ has a basis $\left\{x_{1}, x_{2}\right\}$ with $\left(x_{i}\right)=M\left(g_{i}, \chi_{i}\right)\left(g_{i}\right.$ and $\chi_{i}$ are respectively central elements and characters of a certain subgroup $\Gamma^{\prime}$ of $\left.\Gamma\right)$. Let $N_{i}$ be the order of $\chi_{i}\left(g_{i}\right)$. Then, by 2.2, we have $p^{2} \geq N_{1} N_{2}$, whence $N_{1}=N_{2}=p ;\left(\chi_{i}\left(g_{i}\right) \neq 1\right.$ since $A$ is finite dimensional). Again by Proposition 2.2 we have that $\mathfrak{B}(V)$ is a QLS over $\Gamma^{\prime}$. Let $b_{12}=\chi_{2}\left(g_{1}\right)$, and for each $h \in \Gamma$ let the matrix $\rho(h)_{i j}$ be defined by $h \rightarrow x_{j}=\sum_{i=1}^{2} \rho(h)_{i j} x_{i}$. Then $A$ is generated by $\Gamma, a_{1}, a_{2}$ with structure and relations given by

$$
\begin{gathered}
a_{i}^{p}=0, \quad \varepsilon\left(a_{i}\right)=0, \quad h a_{j} h^{-1}=\sum_{i=1}^{2} \rho(h)_{i j} a_{i}, \quad \forall h \in \Gamma, \\
\Delta\left(a_{i}\right)=g_{i} \otimes a_{i}+1 \otimes a_{i}, \\
a_{1} a_{2}=b_{12} a_{2} a_{1} .
\end{gathered}
$$

4. The bounds $2 \leq \operatorname{dim} V \leq 3$ are immediate consequences of Proposition 2.2. If $\operatorname{dim} V=2$, then by Lemma 4.10 below it is a twisting of an algebra of type $A_{2}$. If $\operatorname{dim} V=3$, then by Proposition 2.2 it is a QLS.

5. As in the case $\Gamma=C_{p} \times C_{p}$, the bounds $2 \leq \operatorname{dim} V \leq 3$ are consequences of Proposition 2.2. Suppose that $\operatorname{dim} V=2, V$ has basis $\left\{x_{1}, x_{2}\right\}$ and $c$ is given in this basis by the matrix $\left(b_{i j}\right)$. If $b_{11}$ (resp. $b_{22}$ ) has order $p^{2}$, then by Proposition $2.2 b_{22}$ (resp. $b_{11}$ ) has order $p$ and $\mathfrak{B}(V)$ is a QLS. If both $b_{11}$ and $b_{22}$ have order $p$ then, by Lemmas 4.9 and 4.10 below, $\mathfrak{B}(V)$ is a twisting of an algebra of type $A_{2}$. If $\operatorname{dim} V=3$, then by Proposition 2.2 it is a QLS.

6. This is proved in [3, Theorem 1.3].

In Section 5 we prove that if $\Gamma$ is a group of order $p^{j}$ and $R=\oplus_{i} R(i) \in \mathbf{k} \Gamma \mathcal{Y} \mathcal{Y} \mathcal{D}$ is a coradically graded braided Hopf algebra of dimension $p^{5-j}$ with $R(1) \simeq \mathbf{k}$, then $R$ is generated by $R(1)$. With this and the previous lemma we can prove the following result.

Theorem 3.2. Let $A=\oplus_{i} A(i)$ be a coradically graded pointed Hopf algebra of dimension $p^{5}$. Let $\Gamma=G(A)$ be the group of group-likes of $A$. Let $R=\bigoplus_{i} R(i)=$ $A^{\mathrm{co} A(0)} \in{ }_{\mathbf{k} \Gamma}^{\mathbf{k} \Gamma \mathcal{D}}$ and let $V=R(1)$. Then $R$ is generated by $V$ (i.e. $A=\mathfrak{B}(V) \# \mathbf{k} \Gamma$ ) and $\mathfrak{B}(V)$ is one in the list below. By $B(\cdot)$ we denote the group of order $p^{4}$ in $[\mathbf{5}$, p. 145]. 


\begin{tabular}{lccl}
\hline$\Gamma$ & $\operatorname{dim} \mathfrak{B}(V)$ & Type & Conditions \\
\hline$\left(C_{p}\right)^{4}$ & 1 & QLS \\
$\left(C_{p}\right)^{2} \times C_{p^{2}}$ & 1 & QLS \\
$C_{p^{2}} \times C_{p^{2}}$ & 1 & QLS \\
$C_{p} \times C_{p^{3}}$ & 1 & QLS \\
$C_{p^{4}}$ & 1 & QLS \\
$B(v i)$ & 1 & QLS \\
$B(v i i)$ & 1 & QLS \\
$B(v i i i)$ & 1 & QLS \\
$B(i x)$ & 1 & QLS \\
$B(x)$ & 1 & QLS \\
$B(x i v)$ & 1 & QLS \\
$\left(C_{p}\right)^{3}$ & 2 & QLS \\
$C_{p^{2}} \times C_{p}$ & 1 & QLS \\
& 2 & QLS \\
$C_{p^{3}}$ & 1 & QLS \\
$\left(C_{p}\right)^{2}$ & 2 & QLS \\
& 2 & $A_{2}$ \\
$C_{p^{2}}$ & 3 & QLS \\
$C_{p}$ & 2 & $A_{2} \quad p=3$ or $p \equiv 1 \bmod 3$ \\
& 2 & $B_{2} \quad p \equiv 1 \bmod 4$ \\
\hline & 3 & $A_{2} \times A_{1} \quad p=3$ \\
\hline
\end{tabular}

Proof. For the groups of order $p^{4}$, the only condition for the existence of a QLS is the existence of a central element $g \in \Gamma$ and a character $\chi \in \hat{\Gamma}$ such that $\chi(g)$ has order $p$. This is possible if and only if $g \notin[\Gamma, \Gamma]$ where $[\Gamma, \Gamma]$ is the commutator subgroup of $\Gamma$. It follows by inspection of each case that the groups in the table are those $\Gamma$ such that $Z(\Gamma) \not \subset[\Gamma, \Gamma]$.

We go now to $|\Gamma|=p^{3}$. It is clear that QLS of rank one exist for $\Gamma=C_{p^{2}} \times C_{p}$ and $\Gamma=C_{p^{3}}$, but not for $\Gamma=\left(C_{p}\right)^{3}$. The two non-abelian groups of order $p^{3}$ have centers included in their commutator subgroups, whence the 1-dimensional YetterDrinfeld modules give rise to infinite dimensional Nichols algebras. We prove now that for the three abelian groups there exist QLS of rank 2: let $q_{1}, q_{2}, q_{3}$ denote respectively (fixed) roots of unity of orders $p, p^{2}, p^{3}$. We denote the generators of $\left(C_{p}\right)^{3}$ by $\left\{g_{1}, g_{2}, g_{3}\right\}$ and the generators of $\left(\widehat{\left.C_{p}\right)^{3}}\right.$ by $\left\{\hat{g}_{1}, \hat{g}_{2}, \hat{g}_{3}\right\}$, where $\hat{g}_{i}\left(g_{j}\right)=q_{1}^{\delta_{i j}}$. We denote the generators of $C_{p^{2}} \times C_{p}$ by $\left\{g_{1}, g_{2}\right\}$ and the generators of $\widehat{C_{p^{2}} \times C_{p}}$ by $\left\{\hat{g}_{1}, \hat{g}_{2}\right\}$, where $\hat{g}_{i}\left(g_{j}\right)=q_{3-i}^{\delta_{i j}}$. We denote the generator of $C_{p^{3}}$ by $\{g\}$ and the generator of $\widehat{C_{p^{3}}}$ by $\{\hat{g}\}$, where $\hat{g}(g)=q_{3}$. It is straightforward that the following Yetter-Drinfeld modules give QLS of dimension $p^{2}$ :

$$
\begin{gathered}
\Gamma=\left(C_{p}\right)^{3}, \quad V=M\left(g_{1}, \hat{g}_{1}\right) \oplus M\left(g_{2}, \hat{g}_{2}\right), \\
\Gamma=C_{p^{2}} \times C_{p}, \quad V=M\left(g_{1}^{p}, \hat{g}_{1}\right) \oplus M\left(g_{1}^{-p}, \hat{g}_{1}\right), \\
\Gamma=C_{p^{3}}, \quad V=M\left(g^{p^{2}}, \hat{g}\right) \oplus M\left(g^{-p^{2}}, \hat{g}\right) .
\end{gathered}
$$


For the two non-abelian groups we should have $V$ a Yetter-Drinfeld module of dimension 2. There are three possibilities.

1. $V=M\left(h_{1}, \chi_{1}\right) \oplus M\left(h_{2}, \chi_{2}\right)$, where $h_{i}$ are central and $\chi_{i}$ are characters; but by the same reason as in the rank one case, this would give infinite dimensional Nichols algebras.

2. $V=M(g, \chi)$, where $\chi$ is a character and $\left[\Gamma: \Gamma_{g}\right]=2$; but this is impossible since $p \neq 2$ (this case arises when $p=2$; see [10]).

3. $V=M(g, \rho)$, where $g$ is central and $\rho$ is an irreducible representation of $\Gamma$ with $\operatorname{deg} \rho=2$. Since $p \neq 2$, by the Frobenius theorem we find that this impossible; (this case arises when $p=2$; see [10]).

Let now $\Gamma=\left(C_{p}\right)^{2}$. It is immediate that there are no QLS of rank 1 nor 2 , since otherwise there would be a character with a $p^{2}$-th root of unity in the image. The existence of a QLS of rank 3 is a consequence of [2, Lemma 4.1]. An explicit construction is as follows: let $\Gamma$ have generators $\left\{g_{1}, g_{2}\right\}$ and $\hat{\Gamma}$ have generators $\left\{\hat{g}_{1}, \hat{g}_{2}\right\}$ where $\hat{g}_{i}\left(g_{j}\right)=q_{1}^{\delta_{i j}}$ (as before $q_{1}$ is a fixed $p$-th root of unity). Let $V=M\left(g_{1}, \hat{g}_{1}\right) \oplus$ $M\left(g_{1}, \hat{g}_{1}{ }^{-1}\right) \oplus M\left(g_{2}, \hat{g}_{2}\right)$. It is straightforward to see that $V$ generates a QLS. For a construction of a Nichols algebra of type $A_{2}$, let $r=\frac{1}{2} \in \mathbb{Z} / p$ (the construction for $p=2$ is slightly different; see [10]). Set $V=M\left(g_{1}, \hat{g}_{1} \hat{g}_{2}{ }^{-r}\right) \oplus M\left(g_{2}, \hat{g}_{1}{ }^{-r} \hat{g}_{2}\right)$. It is clear then that $V$ has the matrix

$$
\left(b_{i j}\right)=\left(\begin{array}{cc}
q_{1} & q_{1}^{-r} \\
q_{1}^{-r} & q_{1}
\end{array}\right) \text {, whence } b_{i j} b_{j i}=b_{i i}^{a_{i j}} \text { with }\left(a_{i j}\right)=\left(\begin{array}{cc}
2 & -1 \\
-1 & 2
\end{array}\right) .
$$

Let $\Gamma=C_{p^{2}}$. The non-existence of a $p^{3}$-dimensional QLS is a consequence of Lemma 4.1 below. Let $g, \hat{g}$ be respectively generators of $\Gamma, \hat{\Gamma}$, and let $q=\hat{g}(g)$. Suppose that $V \in \mathbf{k} \Gamma \mathcal{Y} \mathcal{D}$ generates an algebra of type $A_{2}$. Let $V=M\left(g^{e_{1}}, \hat{g}^{f_{1}}\right) \oplus$ $M\left(g^{e_{2}}, \hat{g}^{f_{2}}\right)$. Since $V$ has a matrix $b_{i j}=q^{e_{i} f_{j}}$ and $b_{11}, b_{22}$ must have order $p$, then $p$ divides $e_{1}$ and $e_{2}$, or $p$ divides $f_{1}$ and $f_{2}$. Then the same arguments as in [3, Theorem 1.3] give the condition $p=3$ or $p \equiv 1 \bmod 3$. Furthermore, let $b$ be such that $b^{2}+b+1 \equiv 0 \bmod p$ (the condition on $p$ is equivalent to the existence of such a $b$ ) and take $e_{1}=p, f_{1}=1$, $e_{2}=-p(b+1), f_{2}=b$. It is straightforward to see that this gives a Cartan matrix of type $A_{2}$.

For $\Gamma=C_{p}$ it is proved in [3, Theorem 1.3] that there exists an algebra of type $B_{2}$ if and only if $p \equiv 1 \bmod 4$, and of type $A_{2} \times A_{1}$ if and only if $p=3$.

4. Subsidiary results. The following lemma may be considered as an addendum to [2, Lemma 4.2].

Lemma 4.1. Let $\Gamma=C_{p^{n}}$ and $V \in{ }_{k \Gamma}^{k \Gamma} \mathcal{Y D}$ generate a finite dimensional $Q L S$. Then $V$ may be 1-dimensional (and hence $\operatorname{dim} \mathfrak{B}(V)=p^{v}$ with $1 \leq v \leq n$ ) or it may be 2dimensional (and hence $\operatorname{dim} \mathfrak{B}(V)=p^{2 v}$ with $1 \leq v \leq n$ ).

Proof. The bound $\operatorname{dim} V \leq 2$ is the content of [2, Lemma 4.2]. Let $\Gamma$ have a generator $g$ and $\hat{\Gamma}$ have a generator $\hat{g}$. Let $q=\hat{g}(g)$, which is a primitive $p^{n}$-th root of unity. If $V$ is 1-dimensional, the result is an easy consequence of Proposition 2.2.

Suppose that $V=M\left(g^{e_{1}^{\prime}}, \hat{g}^{f_{1}^{\prime}}\right) \oplus M\left(g^{e_{2}^{\prime}}, \hat{g}_{2}^{f_{2}^{\prime}}\right)$. Let $e_{i}^{\prime}=p^{r} e_{i}$ such that $e_{1}, e_{2}$ are not both divisible by $p, f_{i}^{\prime}=p^{s} f_{i}$ such that $f_{1}, f_{2}$ are not both divisible by $p$. Then $V$ has a 
matrix given by $b_{i j}=q^{e_{i}^{\prime} f_{j}^{\prime}}=q^{e_{i} f_{j} p^{r+s}}$. Since $\mathfrak{B}(V)$ is finite dimensional, $r+s<n$ (for if not $\left.b_{11}=b_{22}=1\right)$. Let $u=n-r-s$. Suppose that $p \nmid e_{1}$ (if $p \nmid e_{2}$ it is analogous). Suppose first that $p \nmid f_{2}$; then $b_{12}$ has order $p^{u}$. Since $V$ generates a QLS, $b_{21} b_{12}^{-1}$ also has order $p^{u}$ and thus $p \nmid e_{2}, p \nmid f_{1}$. This implies the result with $v=u$. Suppose next that $p \mid f_{2}$. Then $p \nmid f_{1}$. Let $f_{2}=p^{t} b, e_{2}=p^{y} a$ with $p \nmid b, p \nmid a$. We prove that $t=y$ : we have $t<u$ since if not $b_{22}=1$. Now, $b_{12}$ has order $p^{u-t}$, whence $b_{21}$ has order $p^{u-t}$. Since $p \nmid f_{1}$ we have $p^{t} \mid e_{2}$, whence $y \geq t$. By similar considerations $y \leq t$. This implies the result with $v=u-t$.

We shall make use of the following important tool for Nichols algebras.

Definition 4.2. Let $V \in{ }_{H}^{H} \mathcal{Y D}$ and $c=c_{V, V}$. For $i+j=n$, we denote by $\Delta_{i, j}: \mathfrak{B}^{n}(V) \rightarrow \mathfrak{B}^{i}(V) \otimes \mathfrak{B}^{j}(V)$ the $(i, j)$-component of the comultiplication of $\mathfrak{B}(V)$.

It is proved in [14] (or see [1, Definition 3.2.10]) that $\Delta_{i, j}$ is injective, $\forall i, j$. Let $\left\{x_{1}, \ldots, x_{\theta}\right\}$ be a basis of $V$ and let $\left\{x_{1}^{*}, \ldots, x_{\theta}^{*}\right\}$ be its dual basis. We denote by $\partial_{x_{i}}$ the differential operator on $\mathfrak{B}(V)$ given by

$$
\partial_{x_{i}}(z)=\left(\mathrm{id} \otimes x_{i}^{*}\right) \Delta_{n-1,1}(z), \quad \text { if } z \in \mathfrak{B}^{n}(V), n>0, \quad \text { and } \partial_{x_{i}}(1)=0 .
$$

By the injectivity of $\Delta_{i, j}$ it is immediate that for $z \in \mathfrak{B}^{n}(V)(n>0)$ we have $z=0$ if

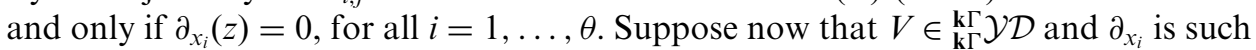
that there exists $g \in \Gamma$ with $\partial_{x_{i}}(v)=0$ if $\delta(v)=h \otimes v$ and $h \neq g$; (this happens for instance if $\delta\left(x_{j}\right)=g_{j} \otimes x_{j}, j=1, \ldots, \theta$ and $\left.g=g_{i}\right)$. Then it is easy to see that $\partial_{x_{i}}$ satisfies the Leibniz rule

$$
\partial_{x_{i}}\left(z_{1} z_{2}\right)=\partial_{x_{i}}\left(z_{1}\right)\left(g \rightarrow z_{2}\right)+z_{1} \partial_{x_{i}}\left(z_{2}\right) .
$$

The following theorem is proved in [2, Theorem 0.2] in the case in which $\Gamma$ is an abelian group.

Theorem 4.3. Let $\Gamma$ be a finite group. Let $V \in{ }_{k \Gamma}^{k \Gamma} \mathcal{Y D}$ be such that $\operatorname{dim} \mathfrak{B}(V)=p^{2}$, where $p$ is the smallest prime number dividing $|\Gamma|$. Then $\operatorname{dim} V \leq 2$ and $\mathfrak{B}(V)$ is a $Q L S$ over some subgroup $\Gamma^{\prime} \subset \Gamma$. Furthermore, if $p>2$ then $V=M(g, \chi)$ with $g$ central, $\chi$ is a character such that $\chi(g)$ has order $p^{2}$ and hence $\mathfrak{B}(V)$ is a $Q L$ over $\Gamma$, or $V=M\left(g_{1}, \chi_{1}\right) \oplus M\left(g_{2}, \chi_{2}\right)$ where $g_{i}$ is central, $\chi_{i}$ is a character $(i=1,2)$ such that $\chi_{i}\left(g_{i}\right)$ has order $p$ and hence $\mathfrak{B}(V)$ is a $Q P$ over $\Gamma$.

Proof. Let $V=\oplus_{i=1}^{\theta} M\left(g_{i}, \rho_{i}\right)$. It can be shown that $\operatorname{dim} \mathfrak{B}(V) \geq \operatorname{dim} \mathfrak{B}\left(M\left(g_{i}, \rho_{i}\right)\right)$, $\forall i$. Let $I=\left[\Gamma: \Gamma_{g_{1}}\right]$ and $d=\operatorname{deg}\left(\rho_{1}\right)$. We have $\operatorname{dim} M\left(g_{1}, \rho_{1}\right)=d I$. We have $d=1$ or $d \geq p$, and $I=1$ or $I \geq p$. Since $\operatorname{dim} \mathfrak{B}(V) \geq 1+\operatorname{dim} V$ we have $\operatorname{dim} V<p^{2}$.

Suppose first that $d \geq p$. This implies that $I=1$, whence $g_{1}$ is central in $\Gamma$. By 2.6 we have $p^{2}=\operatorname{dim} \mathfrak{B}(V) \geq \operatorname{dim} \mathfrak{B}\left(M\left(g_{1}, \rho_{1}\right)\right) \geq N^{d}$ with $N$ the order of $q$, where $q$ id $=\rho_{1}\left(g_{1}\right)$. Since $\mathfrak{B}(V)$ is finite dimensional, we have $q \neq 1$ and hence $N \geq p$. If $p>2$ we have a contradiction. If $p=2$ we must have $\theta=1, d=2, N=2$. The condition $d=2$ implies that $V$ comes from the abelian case, as explained after Definition 2.4. The condition on $N$ tells us that $q=-1$. Furthermore, by Proposition 2.2, $\mathfrak{B}(V)$ is a QLS, and it is shown in [1] that the matrix of $c$ is $\left(\begin{array}{ll}-1 & -1 \\ -1 & -1\end{array}\right)$ 
Suppose then that $I \geq p$. This implies that $d=1$, whence $\rho_{1}$ is a character of $\Gamma_{g_{1}}$. Let $q=\rho_{1}\left(g_{1}\right)$ and let $N$ be the order of $q$. Let $x$ be a generator of the space affording $\rho_{1}$, and let $\left\{h_{1}=1, h_{2}, \ldots, h_{I}\right\}$ be a set of representatives of the cosets of $\Gamma / \Gamma_{g_{1}}$. Then $M\left(g_{1}, \rho_{1}\right)$ has as basis the elements $\left\{h_{1} \rightarrow x, \ldots, h_{I} \rightarrow x\right\}$ and we have

$$
\begin{aligned}
c\left(h_{i} \rightarrow x \otimes h_{i} \rightarrow x\right) & =h_{i} g_{1} h_{i}^{-1} h_{i} \rightarrow x \otimes h_{i} \rightarrow x \\
& =h_{i} g_{1} \rightarrow x \otimes h_{i} \rightarrow x=q\left(h_{i} \rightarrow x \otimes h_{i} \rightarrow x\right) .
\end{aligned}
$$

It is straightforward to see using derivations that the elements

$$
\left\{1,\left(h_{i} \rightarrow x\right)^{r} \mid 1<r<N, \quad i=1, \ldots, I\right\}
$$

are linearly independent, whence

$$
p^{2}=\operatorname{dim} \mathfrak{B}(V) \geq 1+I(N-1) .
$$

Thus, $N \leq p$. On the other hand, $q \neq 1$ for if not it is easy to see using derivations that the elements $\left\{x^{r} \mid r \geq 0\right\}$ would be linearly independent and $\mathfrak{B}(V)$ would be infinite dimensional; (note that we have not proved at present that $\mathbf{k} x$ is a sub-YDmodule nor that $M\left(g_{1}, \rho_{1}\right)$ comes from the abelian case, and hence Proposition 2.2 cannot be used.) We have thus proved that $N=p$. Suppose for a moment that $I>p$. It is clear that if $p>2$ then $I \geq p+2$, but then (4.5) tells us that this is a contradiction. If $p=2$, then $I=3$ but, by [1, Proposition 3.2.2], $\operatorname{dim} \mathfrak{B}\left(M\left(g_{1}, \rho_{1}\right)\right) \geq 5$, also a contradiction. Hence, we have that $I=p$ and then $\Gamma_{g}$, having index the smallest prime dividing $|\Gamma|$, is invariant in $\Gamma$. As stated after Definition 2.4, this implies that $\mathfrak{B}\left(M\left(g_{1}, \rho_{1}\right)\right)$ comes from the abelian case, but then Proposition 2.2 applies and (4.4) tells us that $\operatorname{dim} \mathfrak{B}\left(M\left(g_{1}, \rho_{1}\right)\right) \geq p^{p}$. This is a contradiction if $p>2$. If $p=2$, then $\theta=1, q=-1$ and it is proved in [1] that the matrix of $c$ is

$$
\left(\begin{array}{cc}
-1 & 1 \\
1 & -1
\end{array}\right) \text { or }\left(\begin{array}{ll}
-1 & -1 \\
-1 & -1
\end{array}\right) \text {. }
$$

Suppose finally that $I=d=1$. Then $g_{1}$ is central and $\rho_{1}$ is a character. Let $q=\rho_{1}\left(g_{1}\right)$ and let $N$ be its order. Then $\operatorname{dim} \mathfrak{B}(V) \geq \operatorname{dim} \mathfrak{B}\left(M\left(g_{1}, \rho_{1}\right)\right)=N$ implies that $N \leq p^{2}$. If $N=p^{2}$, then $\theta=1$ and the result follows at once. If $N<p^{2}$, then $N \geq p$ and $N$ is prime. Since $\operatorname{dim} \mathfrak{B}\left(M\left(g_{1}, \rho_{1}\right)\right)=N$, we have $\theta>1$. Since $\operatorname{dim} \mathfrak{B}\left(M\left(g_{2}, \rho_{2}\right)\right) \leq p^{2}-1$ (because if $x$ is a generator of $M\left(g_{1}, \rho_{1}\right)$ then $x$ does not belong to $\left.\mathfrak{B}\left(M\left(g_{2}, \rho_{2}\right)\right)\right)$ by the same arguments as above applied to $M\left(g_{2}, \rho_{2}\right)$ we have necessarily that $g_{2}$ is central and $\rho_{2}$ is a character. Let $N_{2}$ be the order of $\rho_{2}\left(g_{2}\right)$. Thus $N_{2}<p^{2}$, and since $g_{1}, g_{2}$ are both central, $M\left(g_{1}, \rho_{1}\right) \oplus M\left(g_{2}, \rho_{2}\right)$ comes from the abelian case, whence by Proposition 2.2, $\mathfrak{B}\left(M\left(g_{1}, \rho_{1}\right) \oplus M\left(g_{2}, \rho_{2}\right)\right)$ has dimension at least $N N_{2}$. This implies that $N=N_{2}=p, \theta=2$ and $\mathfrak{B}(V)$ is a QLS over $\Gamma$.

REMARK 4.6. It is proved in [8] in a different way that if $\operatorname{dim} \mathfrak{B}(V)=p$, where $p$ is the smallest prime number dividing $\Gamma$, then $\operatorname{dim} V=1$ and $\mathfrak{B}(V)$ is a QLS. It is proved also, with the same ideas as here, in [3, Proposition 7.5].

REMARK 4.7. We note that the proof of Theorem 4.3 above says that there are no $V$ in ${ }_{\mathbf{k} \Gamma}^{\mathrm{k} \Gamma} \mathcal{Y D}$ such that $\operatorname{dim} \mathfrak{B}(V)=\pi^{2}$ if $\pi$ is a prime number smaller than every prime dividing $|\Gamma|$. 
The previous theorem implies the following result.

Corollary 4.8. Let $A$ be a pointed Hopf algebra of dimension $m$ whose coradical has dimension $m / p^{2}$, where $p$ is the smallest prime number dividing $m$. Then $p^{3}$ divides $m$ and $\operatorname{dim} A_{1}=(r+1) m / p^{2}$, where $r=1$ or 2 .

Proof. Consider the coradical filtration of $A$ and let $H=\oplus_{i} H(i)$ be the associated graded algebra. Then $H$ is pointed and $H(0) \simeq \mathbf{k} \Gamma$, where $\Gamma$ is the group of group-likes of $A$ that has order $m / p^{2}$. Let $R=H^{\mathrm{co} H(0)} \in{ }_{\mathbf{k} \Gamma \Gamma}^{\mathbf{k} \Gamma \mathcal{D}}$ and let $R^{\prime} \subset R$ be the algebra generated by $R(1) ;\left(R^{\prime}=R\right.$ if and only if $R$ is a Nichols algebra). Thus $\operatorname{dim} R=p^{2}$ and by the Nichols-Zoeller theorem, $\operatorname{dim} R^{\prime}=p^{j}$ with $0 \leq j \leq 2$. The case $j=0$ would imply that $\operatorname{dim} R(1)=0$, which is impossible. The case $j=1$ is also impossible, for in that case Remark 4.6 says that $\operatorname{dim} R(1)=\operatorname{dim} R^{\prime}(1)=1$, and [2, Theorem 3.2] says that $R$ is a Nichols algebra. Then $R^{\prime}=R$. Remark 4.7 says that $p$ divides $|\Gamma|$ (whence $p^{3}$ divides $m$ ) and Theorem 4.3 says that $r=\operatorname{dim} R(1)$ may be 1 or 2 , whence $\operatorname{dim} H(1)=r|\Gamma|$ and

$$
\operatorname{dim} A_{1}=\operatorname{dim} H(0)+\operatorname{dim} H(1)=(r+1)|\Gamma|=(r+1) m / p^{2} .
$$

LeMmA 4.9. Let $\Gamma$ be a p-group and $V$ a 2-dimensional module in ${ }_{k} \Gamma \mathcal{Y} \mathcal{D}$ such that $\operatorname{dim} \mathfrak{B}(V)=p^{3}$. Recall that under this assumption $c$ has a matrix $\left(b_{i j}\right)$ with respect to some basis $\{x, y\}$. Let $q$ be a primitive $p^{2}$-th root of unity, and suppose that $b_{i j}=q^{c_{i j}}$. If $p$ divides $c_{11}$ and $c_{22}$, then $p$ divides $c_{12}+c_{21}$.

Proof. We have $x^{p}=y^{p}=0$. Let $z=\operatorname{Ad}_{x}(y)=x y-b_{12} y x$ and $\sigma=1-b_{12} b_{21}$. We have

$$
\partial_{x}(z)=b_{12} y-b_{12} y=0, \quad \partial_{y}(z)=x-b_{12} b_{21} x=\sigma x \Rightarrow \partial_{x} \partial_{y}(z)=\sigma .
$$

Furthermore,

$$
\begin{aligned}
\partial_{x}\left(x^{r} y^{s} z^{t}\right) & =(r)_{b_{11}} b_{11}^{t} b_{12}^{s+t} x^{r-1} y^{s} z^{t} \\
\partial_{y}\left(y^{s} z^{t}\right) & =(s)_{b_{22}} b_{21}^{t} b_{22}^{t} y^{s-1} z^{t}+\sum_{i=0}^{t-1} \sigma b_{21}^{i} b_{22}^{i} y^{s} z^{t-1-i} x z^{i}, \\
\partial_{x} \partial_{y}\left(y^{s} z^{t}\right) & =\sigma(t)_{\left(b_{11} b_{12} b_{21} b_{22}\right)} y^{s} z^{t-1} .
\end{aligned}
$$

Thus, if $p \nmid c_{12}+c_{21}$, the order of $\left(b_{11} b_{12} b_{21} b_{22}\right)$ is $p^{2}$, whence the set $\left\{z^{t} \mid 0 \leq t<p^{2}\right\}$ is linearly independent. This implies inductively that the set $\left\{y^{s} z^{t} \mid 0 \leq s<p\right.$, $\left.0 \leq t<p^{2}\right\}$ is linearly independent, and then that the set $\left\{x^{r} y^{s} z^{t} \mid 0 \leq r, s<p\right.$, $\left.0 \leq t<p^{2}\right\}$ is linearly independent, so that $\operatorname{dim} \mathfrak{B}(V) \geq p^{4}$.

The following result is a consequence of [3, Corollary 1.2]. We give a direct proof here.

Lemma 4.10. Let $V=(x, y)$ be a 2-dimensional module in ${ }_{\boldsymbol{k} \Gamma}^{\boldsymbol{k}} \mathcal{Y} \mathcal{D}$ such that $\operatorname{dim} \mathfrak{B}(V)=p^{3}$. Let $V$ have a matrix $\left(b_{i j}\right)$ and suppose that $b_{i j}^{p}=1$, for all $i, j$. Then $b_{i j}$ is a Cartan matrix of type $A_{2}$. 
Proof. Let $q=b_{11}$ and $c_{i j}$ be given by $b_{i j}=q^{c_{i j}}$. We may suppose as above that $b_{12}=b_{21}$. Let $b_{12}=q^{a}, b_{22}=q^{c}$. Take $z=\operatorname{Ad}_{x}(y)=x y-q^{a} y x$, and let $\sigma=1-q^{2 a}$; thus $\sigma \neq 0$, since otherwise $\mathfrak{B}(V)$ would be a QLS and $\operatorname{dim} \mathfrak{B}(V)$ would be $p^{2}$. As before

$$
\partial_{x}(z)=0, \quad \partial_{y}(z)=\sigma x \Rightarrow \partial_{x} \partial_{y}(z)=\sigma
$$

whence

$$
\begin{aligned}
\partial_{x}\left(x^{r} y^{s} z^{t}\right) & =(r)_{q} q^{t+a(s+t)} x^{r-1} y^{s} z^{t} \\
\partial_{y}\left(y^{s} z^{t}\right) & =(s)_{q^{c}} q^{a s+c t} y^{s-1} z^{t}+\sum_{i=0}^{t-1} \sigma q^{(a+c) i} y^{s} z^{t-1-i} x z^{i}, \\
\partial_{x} \partial_{y}\left(y^{s} z^{t}\right) & =\sigma(t)_{q^{1+2 a+c}} y^{s} z^{t-1} .
\end{aligned}
$$

As before, the set $\left\{x^{r} y^{s} z^{t} \mid 0 \leq r, s, t<p\right\}$ is linearly independent; (as a remark, note that we must have $1+2 a+c \not \equiv 0(\bmod p)$ since if not $\mathfrak{B}(V)$ would be infinite dimensional). Now let $w=\operatorname{Ad}_{x}(z)=x z-q^{1+a} z x$. We have

$$
\partial_{x}(w)=0, \quad \partial_{y}(w)=\sigma x^{2}-\sigma q^{1+a} q^{a} x^{2}=\sigma\left(1-q^{2 a+1}\right) x^{2} .
$$

The $(x, y)$-bidegree of $w$ is $(2,1)$, whence the set $\left\{x^{2} y, x z, w\right\}$ must be linearly dependent in order for $\mathfrak{B}(V)$ to be $p^{3}$-dimensional. This implies $2 a+1=0$, which means that $b_{12} b_{21}=b_{11}^{-1}$.

With the same reasoning, we must have $b_{12} b_{21}=b_{22}^{-1}$, and thus $b_{i j} b_{j i}=b_{i i}^{c_{i j}}$ with

$$
c_{i j} \equiv\left(\begin{array}{cc}
2 & -1 \\
-1 & 2
\end{array}\right) \bmod p,
$$

and $b_{i j}$ is a Cartan matrix of type $A_{2}$. As a remark, note that $b_{11}=b_{22}$.

5. The classification is complete. We have to prove that Theorem 3.2 lists all the coradically graded pointed Hopf algebras of dimension $p^{5}$. This amounts to proving that a coradically graded pointed Hopf algebra is generated by its homogeneous component of degree 1, which in turn is equivalent to proving that if $A=\oplus_{i} A(i)$ is a coradically graded pointed Hopf algebra and $R=A^{\mathrm{co} A_{0}}$ is its algebra of coinvariants then $R$ is a Nichols algebra. As in [3, §8], let $S=R^{*}$ be its dual. Then $S$ is a graded braided Hopf algebra in ${ }_{\mathbf{k} \Gamma}^{\mathbf{k}} \mathcal{Y} \mathcal{D}, S=\oplus_{i} S(i)$ and is generated by $S(0) \oplus S(1)$. Furthermore, we have a surjection $S \rightarrow S^{\prime}, S^{\prime}=\mathfrak{B}(S(1))$. We have to prove that $S$ is coradically graded; i.e. that $\mathcal{P}(S)=S(1)$. This is the same as saying that $S^{\prime}=S$. Now, [2, Theorem 3.2] plus Remark 4.6 solve the problem for the cases in which $\Gamma$ has order $p^{4}$ or $p^{3}$, and [3, Theorem 8.2] and [3, Lemma 8.5] solve the problem for the case in which $\Gamma$ has order $p$ or $\Gamma=C_{p} \times C_{p}$. The following theorem solves the pending case.

THEOREM 5.1. Let $\Gamma$ be a finite group and $p$ the smallest prime number dividing $|\Gamma|$. Let $S=\oplus_{i} S(i)$ in ${ }_{k \Gamma} \Gamma \mathcal{Y} \mathcal{D}$ be a graded braided Hopf algebra of dimension $p^{3}$ such that $S(0)=\boldsymbol{k}$ and $S$ is generated by $S(1)$. Suppose that $S(1)$ comes from the abelian 
case; i.e. there exists an abelian subgroup $\Gamma^{\prime} \subset \Gamma$ such that $S(1)$ is a YD-module over $\Gamma^{\prime}$. Then $S$ is a Nichols algebra.

Proof. We prove the statement for $p>2$, the case $p=2$ being treated in [10]. Let $S^{\prime}=\mathfrak{B}(S(1))$, and consider the canonical projection $S \rightarrow S^{\prime}$. We must prove that this is an isomorphism. If $\operatorname{dim} S(1)=3$ then, by Proposition 2.2, we have $\operatorname{dim} S^{\prime} \geq p^{3}$; but this implies that $S^{\prime}=S$ and $S$ is a Nichols algebra. If $\operatorname{dim} S(1)=1$, then [2, Theorem 3.2] shows that $S$ is a Nichols algebra. Hence we are led to consider the case $\operatorname{dim} S(1)=2$. We have $\operatorname{dim} S^{\prime} \leq p^{3}$, and we suppose that $\operatorname{dim} S^{\prime}<p^{3}$. Then by Proposition 2.2 we have $\operatorname{dim} S^{\prime} \geq \bar{p}^{2}$, whence $\operatorname{dim} S^{\prime}=p^{2}$. Now Theorem 4.3 says that $S^{\prime}$ is a QLS over $\Gamma^{\prime}, S^{\prime}(1)$ has a basis $\{x, y\}$ and the braiding $c$ has a matrix $\left(b_{i j}\right)$ in this basis, where $b_{i i}$ are primitive $p$-th roots of unity and $b_{12} b_{21}=1$. Furthermore, the linear spans $\mathbf{k} x$ and $\mathbf{k} y$ are sub-YD-modules over $\Gamma^{\prime}$. Let $z=x_{1} x_{2}-b_{12} x_{2} x_{1} \in S$. If we prove that $z=0$ in $S$, then $\operatorname{dim} S=p^{2}$, but this would be a contradiction and we would be done.

Suppose that $z \neq 0$. Now, it is immediate that $z$ is primitive in $S$. Consider the coradical filtration of $S$ and let $T=\oplus_{i} T(i)$ be the associated graded algebra. We have $x, y, z \in S_{1}$. Consider $\bar{x}, \bar{y}, \bar{z} \in T(1)$. It is easy to see that these elements are linearly independent. We compute the matrix of $c$ for $\{\bar{x}, \bar{y}, \bar{z}\}$. It is given by

$$
\left(b_{i j}^{\prime}\right)=\left(\begin{array}{ccc}
b_{11} & b_{12} & b_{11} b_{12} \\
b_{21} & b_{22} & b_{21} b_{22} \\
b_{11} b_{21} & b_{12} b_{22} & b_{11} b_{12} b_{21} b_{22}
\end{array}\right)=\left(\begin{array}{ccc}
b_{11} & b_{12} & b_{11} b_{12} \\
b_{21} & b_{22} & b_{21} b_{22} \\
b_{11} b_{21} & b_{12} b_{22} & b_{11} b_{22}
\end{array}\right)
$$

Consider now the canonical projection $T \rightarrow T^{\prime}=\mathfrak{B}(T(1))$. Since $\mathbf{k} x$, $\mathbf{k} y$ and $\mathbf{k} z$ are sub-YD-modules of $S$ over $\Gamma^{\prime}$, then $\mathbf{k} \bar{x}, \mathbf{k} \bar{y}$ and $\mathbf{k} \bar{z}$ are sub-YD-modules of $T$ over $\Gamma^{\prime}$. Thus, if $W=(\bar{x}, \bar{y}, \bar{z})$ we have $\operatorname{dim} \mathfrak{B}(W) \leq \operatorname{dim} T^{\prime} \leq \operatorname{dim} T=p^{3}$. Now Proposition 2.2 applies; (notice that $b_{i i}^{\prime}$ has order $p, \forall i$, since $b_{11} b_{22}=1$ would imply that $\operatorname{dim} \mathfrak{B}(W)=\infty)$. Hence $\mathfrak{B}(W)$ is a QLS, but this implies that $1=b_{11}^{2} b_{12} b_{21}=b_{11}^{2}$, which is impossible since $p \neq 2$. Hence, $z=0$ in $S$ and the theorem is proved.

6. Final remarks. In order to give a complete classification of the pointed Hopf algebras of dimension $p^{5}$, the following steps should be taken.

1. For each Nichols algebra $R$ in Theorem 3.2, give all the modules $M$ in ${ }_{\mathbf{k} \Gamma}^{\mathbf{k} \Gamma} \mathcal{Y D}$ such that $\mathfrak{B}(M) \simeq R$.

2. Classify the isomorphism classes of the bosonizations of the Nichols algebras in the previous step; (note that there exist non isomorphic Nichols algebras which give isomorphic algebras after bosonization).

3. For each coradically graded $p^{5}$-dimensional Hopf algebra in the previous step, classify all the liftings.

These steps are highly non trivial. For instance let $\Gamma=C_{p^{n}}$, where $n>0$ and $p \neq 2$, and let $0 \leq s \leq n$. The number of QLS of $\operatorname{rank} 1$ over $\Gamma$ with dimension $p^{s}$ is given by

$$
\sum_{\substack{i+j-n=s \\ i, j \leq n}} \phi(i, j)
$$


while the number of isomorphisms classes of these QLS after bosonization is given by

$$
\sum_{\substack{i+j-n=s \\ i, j \leq n}} \frac{\phi(i, j)}{I(i, j)}
$$

where

$$
\begin{aligned}
\phi(i) & =p^{i-1}(p-1), \\
\phi(i, j) & =\phi(i) \phi(j), \\
I\left(k_{1}, \ldots, k_{r}\right) & =\phi\left(\max \left\{k_{1}, \ldots, k_{r}\right\}\right) .
\end{aligned}
$$

Furthermore, the number of QLS of rank 2 over $\Gamma$ with dimension $p^{s}$, (where $s$ is even, by Lemma 4.1), is given by

$$
\frac{1}{2} \sum_{i_{1}, j_{1}, i_{2}, j_{2}} \frac{\phi\left(i_{1}, j_{1}\right) \phi\left(i_{2}, j_{2}\right)}{L_{n}\left(i_{1}, j_{2}\right)},
$$

while the number of isomorphism classes of these QLS after bosonization is given by

$$
\frac{1}{2} \sum_{i_{1}, j_{1}, i_{2}, j_{2}} \frac{\phi\left(i_{1}, j_{1}\right) \phi\left(i_{2}, j_{2}\right)}{L_{n}\left(i_{1}, j_{2}\right) I\left(i_{1}, i_{2}, j_{1}, j_{2}\right)},
$$

where

$$
L_{n}(i, j)=\phi(i+j-n)
$$

and the sum is over the tuples such that

$$
\begin{gathered}
i_{1}+j_{1}-n=s_{1} \geq 1, \quad i_{2}+j_{2}-n=s_{2} \geq 1, \\
s_{1}+s_{2}=s, \quad i_{1}, j_{1}, i_{2}, j_{2} \leq n, \quad i_{1}+j_{2}=i_{2}+j_{1} .
\end{gathered}
$$

As a result of this, the number of coradically graded non isomorphic Hopf algebras of dimension $p^{5}$ with coradical $C_{p^{4}}, C_{p^{3}}$ is, respectively, 2( $\left.p^{2}-1\right)$ and $p(p-1)\left[2+\frac{p(p-1)(p+2)}{2}\right]$.

See also the discussion in $[3, \S 9]$ for the first step, $[3, \S 6]$ for the second. In particular, a necessary and sufficient condition for two YD-modules to give isomorphic algebras after bosonization is given in [3, Proposition 6.3].

As an example of the last step, let $A$ be a pointed Hopf algebra of dimension $p^{5}$ with coradical $\Gamma$ of order $p^{4}$. Let $H$ be the associated graded Hopf algebra and $R$ its invariants as in (2.1). Then $H=R \# \mathbf{k} \Gamma$, where $R=\mathfrak{B}(V)$. We have then $V=M(h, \chi)$ where $h$ is central and $\chi$ is a character such that $\chi(h)$ has order $p$. Let $x$ be a generator of $V$. Then, by [10, Proposition 2.0.17], $x$ can be lifted to $a \in A$ such that $\Delta(a)=h \otimes a+a \otimes 1$ and $g a g^{-1}=\chi(g) a \forall g \in \Gamma$. Since the elements $\left\{x^{i} \# g \mid 0 \leq i<p\right.$, $g \in \Gamma\}$ are a basis of $H$, the elements $\left\{a^{i} g \mid 0 \leq i<p, g \in \Gamma\right\}$ are a basis of $A$. The lifting $A$ is then determined by the element $a^{p}$, the case $a^{p}=0$ being the bosonization 
$A=R \# \mathbf{k} \Gamma$. It is easy to see that $a^{p}$ is a skew-primitive and $\Delta\left(a^{p}\right)=h^{p} \otimes a^{p}+a^{p} \otimes 1$. Looking at the space of skew-primitives, this implies that

$$
a^{p}=\lambda\left(h^{p}-1\right), \quad \lambda \in \mathbf{k} .
$$

Taking a suitable scalar multiple of $a$ we may suppose that $\lambda \in\{0,1\}$. Hence there are no more than 2 liftings. In some of the cases we must have $a^{p}=0$. These cases are given by the diamond lemma

$$
\begin{aligned}
& g a^{p}=g \lambda\left(h^{p}-1\right)=\lambda\left(h^{p}-1\right) g \\
& g a^{p}=\chi(g) a g a^{p-1}=\ldots=\chi^{p}(g) a^{p} g=\chi^{p}(g) \lambda\left(h^{p}-1\right) g
\end{aligned}
$$

whence $\lambda\left(\chi^{p}-1\right)=0$ for $A$ to be $p^{5}$-dimensional. This tells us that over the group $B(v i)$ any pointed Hopf algebra of dimension $p^{5}$ is coradically graded.

On the other hand, it is clear that if $h^{p}=1$ then $a^{p}=0$. This tells us that over the groups $B(v i i i), B(i x), B(x)$ and $B(x i v)$ any pointed Hopf algebra of dimension $p^{5}$ is coradically graded.

As a corollary we note that a pointed Hopf algebra of dimension $p^{5}$ and non abelian coradical is coradically graded, unless its coradical is isomorphic to $\mathbf{k} B(v i i)$. We classify all the liftings in this case: $B(v i i)$ can be presented with generators $X, Y, Z$ and relations

$$
X^{p^{2}}=Y^{p}=Z^{p}=1, \quad[Z, Y]=X^{p}, \quad[X, Y]=[X, Z]=1 .
$$

Hence $Z(B(v i i))=(X)$ while $[B(v i i), B(v i i)]=\left(X^{p}\right)$. Let $q$ be a (fixed) $p$-th root of unity. The Yetter-Drinfeld modules generating Nichols algebras of dimension $p$ are then

$$
V=M\left(X^{i}, \chi\right) \text { such that } p \nmid i, \chi(X)=q^{a}(p \nmid a), \chi(Y)=q^{b}, \chi(Z)=q^{c} .
$$

However, it can be shown that most of them give isomorphic algebras after bosonization. We are led to consider two modules:

$$
\begin{gathered}
V_{i}=M\left(X, \chi_{i}\right)(i=1,2), \quad \chi_{1}(X)=q, \quad \chi_{1}(Y)=\chi_{1}(Z)=1, \\
\chi_{2}(X)=\chi_{2}(Y)=q, \quad \chi_{2}(Z)=1 .
\end{gathered}
$$

Hence we have two pointed Hopf algebras of dimension $p^{5}$ with non abelian coradical that are not coradically graded:

$A^{(1)}$ generated by $a, X, Y, Z$ and the relations of $B(v i i)$ together with $X a=q a X, Y a=a Y, Z a=a Z, a^{p}=X^{p}-1, \Delta(a)=X \otimes a+a \otimes 1$,

$A^{(2)}$ generated by $a, X, Y, Z$ and the relations of $B(v i i)$ together with $X a=q a X, Y a=q a Y, Z a=a Z, a^{p}=X^{p}-1, \Delta(a)=X \otimes a+a \otimes 1$.

A description of the liftings of QLS (respectively of the algebras of type $A_{2}$ over groups of exponent $p$ ) is made in [2] (respectively [4]). 


\section{REFERENCES}

1. N. Andruskiewitsch and M. Graña, Braided Hopf algebras over non abelian finite groups, q-alg 9802074, Boletín de la Acad. Nac. Cs. Córdoba, Argentina, 63 (1999), 45-78.

2. N. Andruskiewitsch and H.-J. Schneider, Lifting of quantum linear spaces and pointed Hopf algebras of order $p^{3}$, J. Algebra 209 (1998), 659-691.

3. N. Andruskiewitsch and H.-J. Schneider, Finite quantum groups and Cartan matrices, Adv. Math., to appear.

4. N. Andruskiewitsch and H.-J. Schneider, Lifting of Nichols algebras of type $A_{2}$ and Pointed Hopf algebras of order $p^{4}$, in Hopf algebras and quantum groups, Proceedings of the Colloquium in Brussels 1998, ed. S. Caenepeel, (1999), 1-18.

5. W. Burnside, Theory of groups of finite order, 2nd edition (Dover Publications, New York, 1955).

6. M. Beattie, S. Dăscălescu and L. Grünenfelder, On the number of types of finitedimensional Hopf algebras, Inventiones Math., 136 (1999), 1-7.

7. S. Caenepeel, S. Dăscălescu and S. Raianu, Classifying pointed Hopf algebras of dimension 16, Comm. Algebra, to appear.

8. S. Dăscălescu, Pointed Hopf algebras with large coradical, Comm. Algebra 27 (1999), No. $10,4827-4851$.

9. S. Gelaki, On pointed Hopf algebras and Kaplansky's tenth conjecture, J. Algebra 209 (1998), 635-657.

10. M. Graña, Pointed Hopf algebras of dimension 32, Comm. Algebra 28 (2000), No. 6, 2935-2976.

11. S. Montgomery, Hopf algebras and their actions on rings, CMBS 82 (Amer. Math. Soc., 1993).

12. W. D. Nichols, Bialgebras of type one, Comm. Algebra 6 (1978), 1521-1552.

13. M. E. Sweedler, Hopf algebras (Benjamin, New York, 1969).

14. P. Schauenburg, A characterization of the Borel-like subalgebras of quantum enveloping algebras, Comm. Algebra 24 (1996), 2811-2823. 